\title{
Original stakes and behavior in the prisoner's dilemma game
}

\section{DANIEL ARANOFF AND JAMES T. TEDESCHI, UNIVERSITY OF MIAMI, Coral Gables, Florida}

A 5 by 2 by 2 design was used to investigate the effects in the $P D G$ of giving an advantage to one of the players of an original stake of $0,25,50,100$, or 500 points under two levels of conflict intensity. The $216 \mathrm{Ss}$ were assigned to like-sex dyads. The results showed that the 25 point $O S$ Ss cooperated more and as dyads produced fewer $D D$ outcomes than the other OS Ss. The high conflict condition produced more defections, more DD outcomes, fewer CC outcomes, and fewer CD outcomes than the low conflict condition. Finally, female dyads had more CC outcomes than males.

In many bargaining situations, one party has more resources to spend than the other. If we assume that there is no difference between the two parties as to how willing each is to spend his resources for bargaining objectives, the question arises as to how the original advantage in magnitude of resources affects the bargaining process. Is the "powerful" party more likely to be conservative and is the disadvantaged player more likely to take risks in his decisions? The present study of tacit bargaining with the Prisoner's Dilemma Game (PDG) investigated whether an original stake (OS) of points as an initial advantage to one player will affect the strategy selections of either player.

Five levels of original stakes were studied: In Group 1 neither S was given an OS. In Groups 2, 3, 4, and 5, respectively, Ss were given OSs of $25,50,100$ and 500 points. The two matrices which were employed to represent different conflict intensity levels (Steele \& Tedeschi, 1967) are shown in Fig. 1. Based on previous findings (Jones et al, 1968; Tedeschi et al, in press) it was hypothesized that the highest intensity of conflict would "wash out" the effects of original stakes. The distribution of Ss by sex over the experimental conditions completed the 5 by 2 by 2 design.

Method

A total of $216 \mathrm{Ss}, 100$ males and 116 females, who were fulfilling a course requirement by their participation, were drawn from the introductory psychology courses at the University of Miami.

Ss were met by the $\mathrm{E}$ in like-sex pairs. Each $\mathrm{S}$ drew from a deck of 52 playing cards, with the high draw gaining the original stake. Ss were then seated before game boards in separate rooms. The game board consisted of two switches at its base, a plastic display of the matrix at the top center of the board's face (the cells of which lighted up after each trial to give $S$ feedback about trial outcomes), and two cumulative counters on which were registered the points of both players and which were clearly labeled "yours" and "others." 1 ss were left alone to read Ditto copies of the instructions. When Ss were observed through a one-way mirror to be no longer attending to the instructions, E re-entered the room, answered questions, and left to monitor play from a separate room. The instructions made clear the relationship between mutual strategy selections and matrix outcomes as well as pointing out the OS advantage in the experimental groups. Ss were instructed to gain as many points as they could during the course of the experiment.

After 10 practice trials, the counters were set with the OS values and the 200 experimental trials were begun.

Results

A 2 by 5 by 2 by 2 multivariate analysis of variance tested the effects of sex, OS, matrices, and advantaged vs disadvantaged players. In the control condition, one $S$ from each dyad was randomly assigned to one or the other position for the purpose of data analysis. It was found that there were main effects of $O S$ $(F=2.365 ; \quad d f=4 / 176 ; \quad p<.05)$ and matrices $(F=46.128$; $\mathrm{df}=1 / 176 ; \mathrm{p}<.01$ ) on the overall proportion of defection strategy selections by Ss. No other main effects or interactions were significant. Assuming that Ss are not independent, the
LOW INTENSITY CONFLICT

HIGH INTENSITY CONFLICT

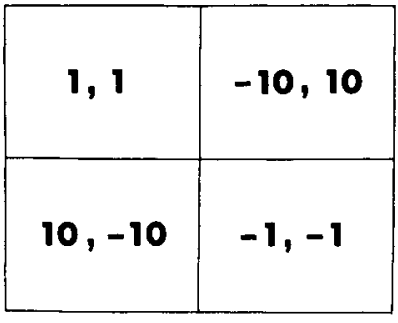

MAIRIX I

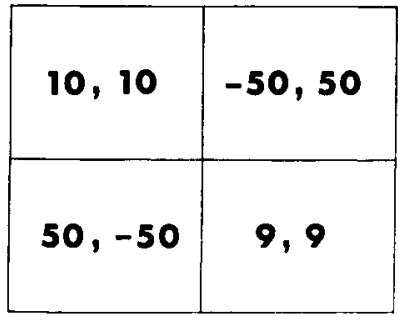

MATRIX 2
Fig. 1. The two matrices used in the experiment.

advantage-disadvantage dimension was collapsed. The main effects of both OS and matrices were still obtained.

Scheffe tests showed that dyads in the 25-point OS condition cooperated more than $S s$ in the other groups and that the 500-point OS Ss defected more than the control Ss. A further analysis showed that $O S$ conditions yielded a linear trend $(\mathrm{F}=3.522 ; \mathrm{df}=1 / 88 ; \mathrm{p}<.04)$ with defection strategy selections. Figure 2 shows that most of the linear effect is contributed by the high conflict matrix and that the latter yields more overall defections at all stake levels than the low conflict matrix.

The dyadic outcomes, CC, DD, and CD, were also analyzed. CC outcomes were extremely rare, occurring only $2.73 \%$ of the time. However, this dependent variable was sensitive to the main effects of $\operatorname{sex}(F=3.872 ; \mathrm{df}=1 / 88 ; \mathrm{p}<.05)$ and matrices $(\mathrm{F}=19.573$; $\mathrm{df}=1 / 88 ; \mathrm{p}<.001)$. A significant linear trend between $\mathrm{OS}$ and CC outcomes was also obtained $(F=5.387 ; \mathrm{df}=1 / 88 ; \mathrm{p}<.02)$. The latter effect is shown in Fig. 3. Women had more CC outcomes than men and the high conflict matrix produced fewer CC outcomes than the low conflict matrix.

There were significant main effects of $O S(F=2.395 ; \mathrm{df}=4 / 88$; $\mathrm{p}<.05)$ and matrices $(\mathrm{F}=47.428 ; \mathrm{df}=1 / 88 ; \mathrm{p}<.001)$ on $D D$ outcomes. Ss in the 25-point OS condition had fewer DD outcomes than Ss in the other four groups. The high conflict matrix yielded more DD outcomes than the low conflict matrix.

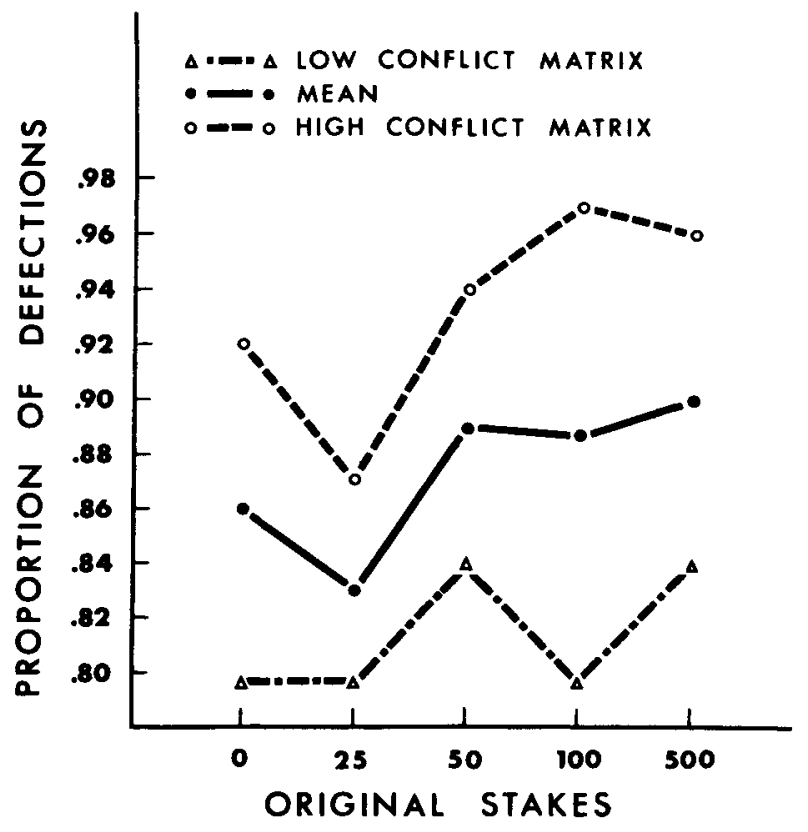

Fig. 2. The effects of OS on defections by matrix condition. 


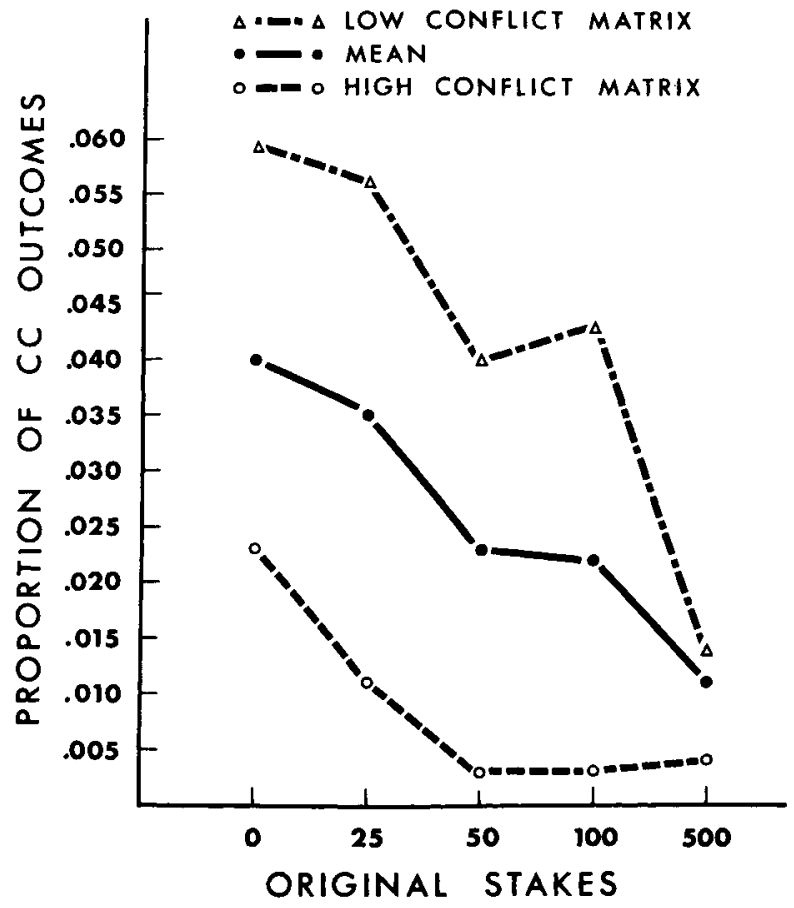

Fig. 3. The effects of OS on $\mathrm{CC}$ outcomes by matrix condition.

Only the main effect of matrices was evident on the $C D$ variable $(\mathrm{F}=44.608 ; \mathrm{df}=1 / 88 ; \mathrm{p}<.001)$ with the high conflict matrix yielding fewer unstable outcomes.

Discussion

The results clearly show that players with the advantage of an OS do not play the PDG differently from those disadvantaged. Consequently, it must be concluded that risk-taking is not associated with the differential amounts of resources of the players in the PDG. On the other hand, it is also clear that a small OS ( 25 points) does induce more cooperation by both players and fewer DD dyadic outcomes than the larger OS conditions or the control situation lacking original advantage to one of the players. Only an interaction hypothesis can explain these results.

In the control condition each player chooses competitively on the average of $86 \%$ of the time. Even higher competitive rates occur in the OS conditions of 50,100, and 500 points. The linear trends obtained from the data demonstrate these effects. Apparently, in all conditions except for the OS condition of 25 points $S$ s perceive the situation in terms of competitive advantage and increasingly so as the $O S$ advantage for one of the players increases. Thus, the player with the OS defends his advantage by using a minimax strategy while the disadvantaged player attempts exploitation by use of the same strategy.

In the 25-point OS condition, the player with an advantage, particularly in the high conflict matrix condition, must have perceived his advantage as minimal since it could be easily removed by one or several plays of the PDG. The disadvantaged player when taking the unilateral initiative to cooperate had a better chance of inducing reciprocation on the part of the other because cooperation was more believable as "rational" strategy and less likely to be used as an attempt to draw the advantaged player into an exploitation. The interaction between the players in the small OS condition thus led to more cooperations and fewer DD outcomes.

The results showing that the high conflict matrix induced more DD outcomes as well as fewer CC and CD outcomes than the low conflict matrix are consistent with the interpretation that the index of matrix values, $\log (\mathrm{T}-\mathrm{S}) /(\mathrm{R}-\mathrm{P})$, developed by Steele \& Tedeschi (1967) is a measure of conflict intensity.

Although female dyads produced more CC outcomes than males, contrary to the results found by Rapoport \& Chammah (1965), the fact that more of the difference is attributable to the low conflict condition conforms to the hypothesis of Jones, Steele, Gahagan \& Tedeschi (1968) that sex differences are obtained in the PDG only under low conflict conditions.

\section{REFERENCES}

JONES, B., STEELE, M., GAHAGAN, J., \& TEDESCHI, J. Matrix values and cooperative behavior in the Prisoner's Dilemma Game. J. Pers. soc. Psychol, 1968, 8, 148-153.

OSGOOD, C. An alternative to war or surrender. Urbana: University of Illinois Press, 1962.

RAPOPORT, A., \& CHAMMAH, A. M. Sex differences in factors contributing to the level of cooperation in the prisoner's dilemma game. J. Pers. soc. Psychol., 1965, 2, 831-838.

STEELE, M. W., \& TEDESCHI, J. T. Matrix indices and strategy choices in mixed-motive games. J. conflict Resolut., 1967, 11, 198-205.

TEDESCHI, J., LESNICK, S., \& GAHAGAN, J. Feedback and "wash-out" effects in the prisoner's dilemma game. J. Pers. soc. Psychol., (in press). NOTE

1. A more complete description of the apparatus and general procedure can be found in Tedeschi, Lesnick, \& Gahagan (in press). 